\title{
XMM-Newton view on cluster outskirts
}

\author{
Alexis Finoguenov \\ Max-Planck-Institut für extraterrestrische Physik, Giessenbachstraße, 85748 Garching, \\ Germany email: alexis@mpe.mpg.de
}

\begin{abstract}
We present an overview on the state of the gas in cluster outskirts. We show that the entropy of the gas in the outskirts often exhibit strong deviations from predictions based on the cluster scaling. We identify the cause of these deviations with incomplete shock propagation observed in clusters with ongoing major merging, survival of substructure and incomplete thermalization, and emission from the surrounding large-scale structure seen in projection.
\end{abstract}

\section{Introduction}

X-ray observations of outskirts of groups and clusters of galaxies are largely driven by an interest to the gravitational mass measurement out to the virial radius. Modelling the state of the gas in cluster outskirts implies a dominant role of gravitational heating, suggesting a simpler picture with respect to cluster centers, where entropy levels are much lower and are affected by various feedback effects, such as preheating and cooling.

Analysis of combined ROSAT and ASCA data on the entropy profiles for groups revealed a very high entropy level in the outskirts, up to $400 \mathrm{keV} \mathrm{cm}{ }^{2}$ (Finoguenov et al. 2002). After comparison with a larger sample of groups and clusters (Finoguenov et al. $2000,2001)$, it was shown that there is a variation in the entropy $\left(k T_{e} / n_{e}^{2 / 3}\right)$ between $\sim 100 \mathrm{keV} \mathrm{cm}{ }^{2}$ and $\sim 400 \mathrm{keV} \mathrm{cm}^{2}$ within every system due to non-gravitational heating. The observed entropy levels are extremely high in the outer parts of groups and this suggests that there has been an energy release into a low-density environment (Finoguenov et al. 2002). In Finoguenov et al. (2003a) we introduce such a phenomenological model for the spatial distribution of the feeback into the GADGET code and were able to recover the observed entropy profiles from groups to clusters of galaxies. The entropy can be further increased by subsequent shock heating associated with cluster formation (Ponman, Sanderson, Finoguenov 2003; Voit et al. 2003).

Thus, these new observations revealed the crucial importance of cluster outskirts in understanding the state of the gas prior to its accretion to the cluster. In this contribution, we have picked out various examples on how the process of cluster formation is recorded in the entropy of the gas located in cluster outskirts.

\section{RGH 80 / NRGs241}

This group is selected from a sample of Ramella-Geller groups detected in X-rays, as cataloged in Mahdavi et al. (2000) and followed up with XMM-Newton (Finoguenov et al., Mahdavi et al., in preparation).

Fig. 1 shows a clear condensation in both the X-ray and the optical to the south. Fig. 1 also displays a velocity histogram of the group. It is double-peaked, and the high-redshift peak corresponds to the southern condensation. The entropy map shows that both the central clump and the southern clump are embedded in a low-entropy zone. The pressure map includes the southern clump, and we can show that it is more likely that the pressure enhancement corresponds to a separate dark matter blob, associated with the potential 

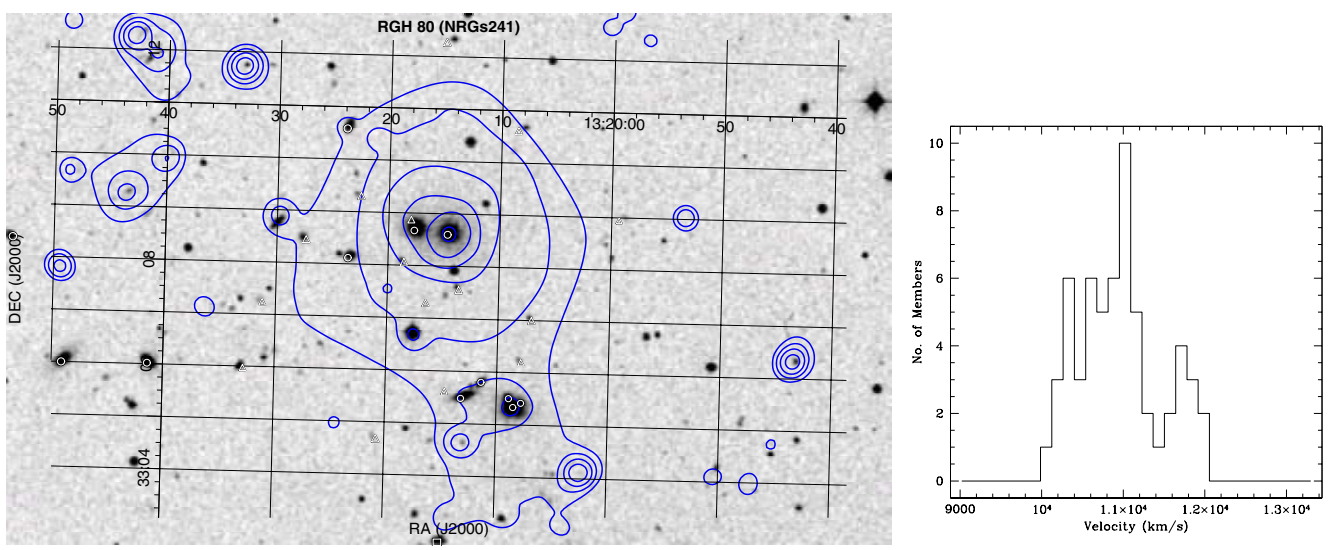

Figure 1. Left panel: optical (B-band) image of the RGH 80 with X-ray contours overlaid. Right panel: velocity histogram of the group.

of the infalling group, and less likely that it corresponds to a shock. The spectral fits shows that there are two components, which are consistent with being projected on top of each other. In the northern source, we observe a rise of the temperature from $0.9 \mathrm{keV}$ to $1.2 \mathrm{keV}$, and then in the outer regions back down again to $0.7 \mathrm{keV}$. The metallicity is enhanced in the southern peak compared to the rest of the image - it is distinct from its surroundings. So, while the infalling group exhibits a low-entropy tail, indicative of a ram-pressure stripping, little disturbance is seen in the main group, indicating that the infalling group has a separation along the line-of-sight. The observed difference in the velocities between the groups is sufficient for the stripping to take place even in the outskirts of the group.

\section{XMM cluster mosaics}

XMM-Newton obtained a number of cluster mosaics as a part of the GTO program of the XMM telescope scientist. Analysis of the mosaics on A3667, A754, A3562, A3266, A2256 is completed (Briel et al. 2004; Henry et al. 2004; Finoguenov et al. 2004c; Jones et al., in preparation; Briel et al., in preparation). Advantages of mosaicing for cluster outskirts are many, in particular better control over the instrumental background that could be achieved; larger radii of the cluster that could be covered. Coma cluster takes a special place in cluster mosaics as the best-covered nearby cluster, yielding a rich collection of publications (Briel et al. 2001; Arnaud et al. 2001; Neumann et al. 2001; Neumann et al. 2003; Finoguenov et al. 2003b; Finoguenov et al. 2004a; Finoguenov \& Miniati 2004; Schuecker et al. 2004).

For the purpose of this presentation we have selected three points clearly seen with these data: incomplete shock propagation, observed in A754; intra-supercluster medium observed in A3562 and projected emission from filaments, observed in the Coma cluster.

\subsection{A754}

Explanation of the observed properties of the A754 core has long been known to require a major merger (Roettiger et al. 1998). As a part of this process, one expects to see a forward shock propagating outward from the cluster center. Krivonos et al. (2001) has detected a sharp edge in the ROSAT data almost half a degree east from the A754 center. Our data provide a temperature measurement across the ROSAT edge, clearly revealing that the edge is a shock with a Mach number of $1.9 \pm 0.4$ and a temperature jump from 

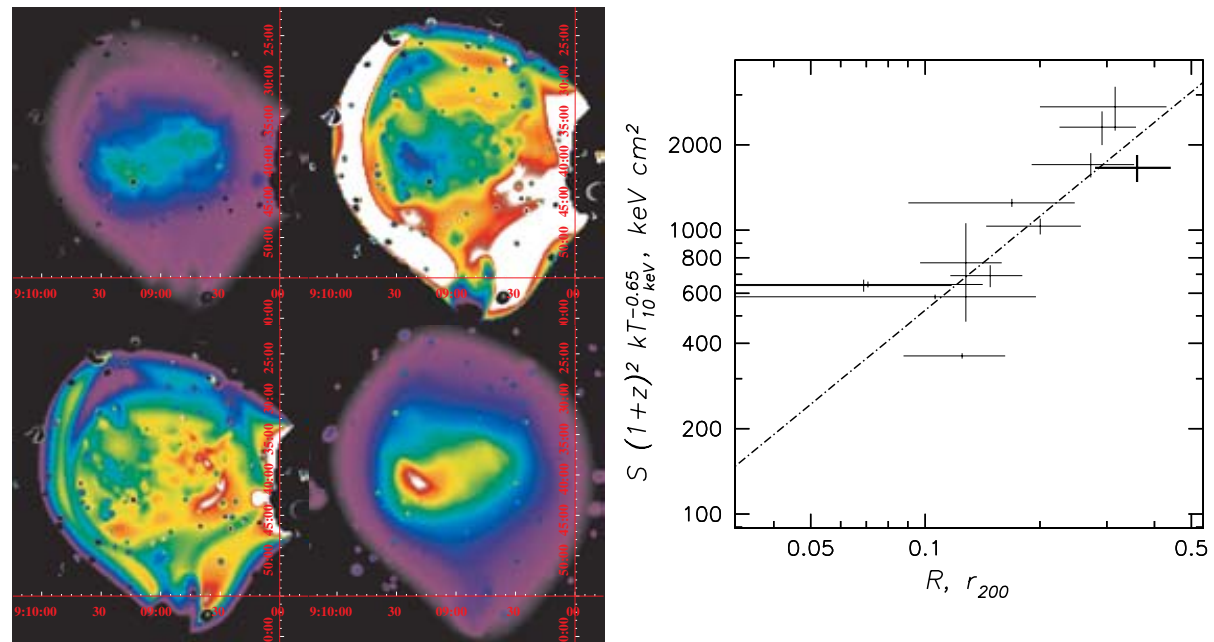

Figure 2. Left panel: pressure, entropy, temperature and surface brightness maps of A754 (from upper left to lower right). Right panel: scaled entropy profile of A754. The universal entropy scaling relation of Ponman et al. (2003) is shown as a dot-dashed line. Bold cross represents the pre-shock state of the gas in the outskirts of A754.

$5.4 \pm 0.6 \mathrm{keV}$ to $9.6 \pm 0.3 \mathrm{keV}$. As shown in Fig. 2, the material preceding the shock reveals a lower entropy compared to adjacent regions closer to the cluster center. Thus, the presence of low temperature, low entropy gas in the outskirts of clusters could simply reflect the state of the cluster before the cluster merger.

\subsection{A3562}

Using the mosaic of six XMM-Newton observations, we study the hydrodynamic state of A3562, a cluster in the center of the Shapley supercluster. The X-ray image reveals a sharp surface brightness gradient within the core of A3562, a $200 \mathrm{kpc}$ ridge extending to the south-west. A nearby group, SC1329-313, imaged within this program also exhibits a tail aligned with the ridge. Study of the pressure and entropy identified the ridge with a $40 \%$ pressure enhancement. An associated Mach number of 1.15 relative to a polytropic sound speed for a $5 \mathrm{keV}$ plasma requires the velocity of the group to be $1400 \mathrm{~km} / \mathrm{s}$, while the projected velocity difference between the cluster and the group amounts to 1200-1500 $\mathrm{km} / \mathrm{s}$. Apparent disruption of the group, if attributed to the ram pressure, also requires the velocity of the group in the cluster frame to amount to $1700 \pm 150 \mathrm{~km} / \mathrm{s}$. The sharp surface brightness gradient at the center is identified with a contact discontinuity, which, together with dove tails in the the large-scale entropy distribution, reveals a sloshing of the BCG in response to the passage of the SC1329-313 group. By using the extent of the low-entropy tails in A3562 we estimate the amplitude of the sloshing motion to be $200 h_{70}^{-1} \mathrm{kpc}$ and the oscillation frequency 1 Gyr.

A filamentary X-ray structure on a several degree scale was reported by ROSAT (Kull \& Böhringer 1999). In order to provide some insights into the origin of the large-scale emission, we have constructed two regions, which reach $25^{\prime}$ distance from the A3562 center. Background subtraction becomes an issue for these regions and we have tried several background accumulations to test the robustness of our results. In addition to the level of instrumental background, as revealed by the data in the $5-12 \mathrm{keV}$ band, we find a need to include an additional $0.2 \mathrm{keV}$ component with solar element abundance typical to 

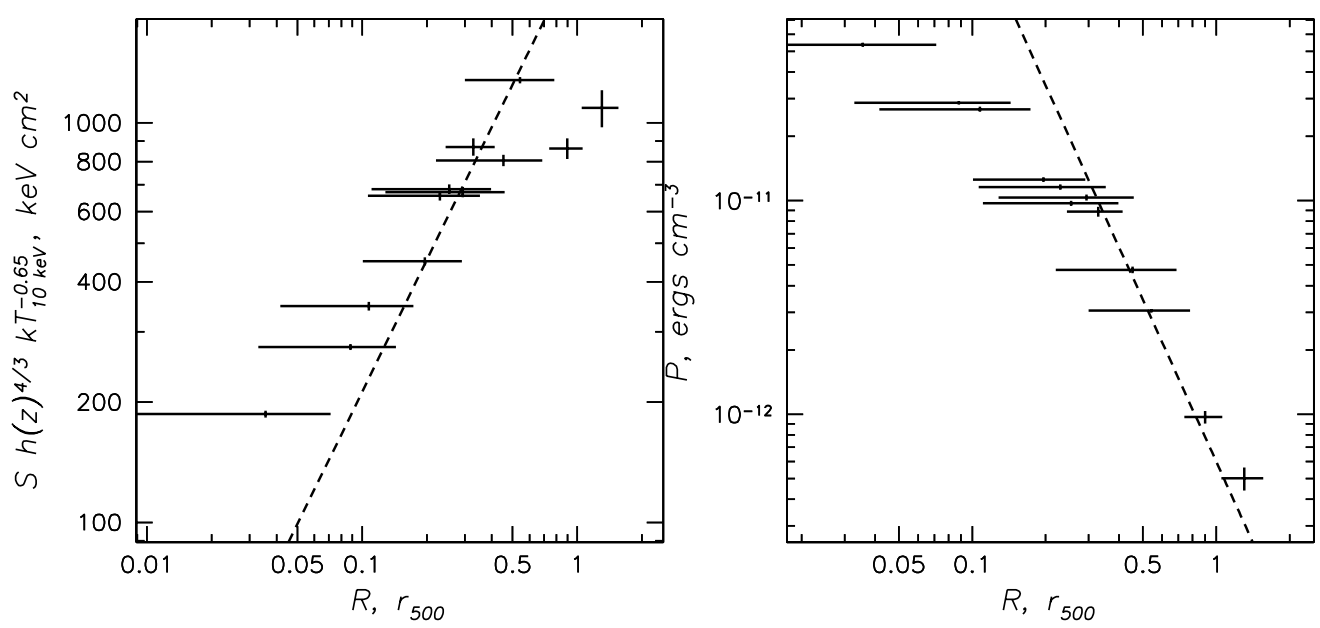

Figure 3. Left panel: entropy profile of the main zones in A3562. Universal entropy scaling relation of Ponman et al. (2003) is shown with a dashed line.Right panel: pressure profile of the main zones in A3562. A pressure slope of -2.5, typical of relaxed clusters, is shown with a dashed line. Normalization is done using the outmost point.

galactic halo emission. The main component, attributed to the Shapley supercluster, has a temperature of $2 \mathrm{keV}$, which is colder compared to the $3 \mathrm{keV}$ temperature observed on the western part of A3562. The state of the gas in the outskirts of A3562, shown in Fig. 3 is characteristic of virial regions of clusters with already maintained pressure balance, but the lower entropy level indicates either a survival of low-entropy gas or an incomplete thermalization.

\subsection{Coma}

XMM-Newton observations of the outskirts of the Coma cluster of galaxies confirm the existence of warm X-ray gas claimed previously and provide a robust estimate of its temperature $(\sim 0.2 \mathrm{keV})$ and oxygen abundance $(\sim 0.1$ solar $)$. Associating this emission with a $20 \mathrm{Mpc}$ infall region in front of Coma, seen in the skewness of its galaxy velocity distribution, yields an estimate of the density of the warm gas of $\sim 50 \rho_{\text {critical }}$. Our measurements of gas mass associated with the warm emission strongly support its nonvirialized nature, suggesting that we are observing the warm-hot intergalactic medium (WHIM). Our measurements provide a direct estimate of the $\mathrm{O}, \mathrm{Ne}$ and Fe abundance of the WHIM. Differences with the reported Ne/O ratio for some OVI absorbers hint to different origins of the OVI absorbers and the Coma filament. Further details on this work could be found in Finoguenov et al. (2003).

\section{Acknowledgements}

The XMM-Newton project is supported by the Bundesministerium für Bildung und Forschung/Deutsches Zentrum für Luft- und Raumfahrt (BMBF/DLR), the Max-PlanckGesellschaft (MPG) and the Heidenhain-Stiftung, and also by PPARC, CEA, CNES, and ASI. AF acknowledges support from BMBF/DLR under grant 50 OR 0207 and MPG. AF thanks his collaborators on various projects reported in this contribution, in particular Uli Briel, Pat Henry, Hans Böhringer, Andisheh Mahdavi, and Mark Henriksen. It is a pleasure to thank Antonaldo Diaferio for organizing such a productive meeting. 


\section{References}

Arnaud, M., Aghanim, N., Gastaud, R., et al. 2001, A\&A, 365, L67

Briel U.G., Henry J.P., Lumb D.H., et al. 2001, A\&A, 365, L60

Briel, U.G., Finoguenov, A., Henry, J.P. 2003, A\&A, submitted

Finoguenov, A., David, L. P., Ponman, T. J. 2000, ApJ, 544, 188

Finoguenov, A., Arnaud, M., David, L.P. 2001, ApJ, 555, 191

Finoguenov, A., Jones, C., Böhringer, H., Ponman, T.J. 2002, ApJ, 578, 74

Finoguenov, A., Borgani, S., Tornatore, L., Böhringer, H. 2003a, A\&A, 398, L35

Finoguenov, A., Briel, U.G. and Henry, J.P. 2003b, A\&A, 410, 777

Finoguenov, A., Briel, U.G., Henry, P.J., et al. 2004a, A\&A, in press [arXiv:astro-ph/0403216]

Finoguenov, A., Miniati, F. 2004, A\&A, 418, L21

Finoguenov, A., Henriksen, M.J., Briel, U.G., et al. 2004b, A\&A, submitted

Henry, P.J., Finoguenov, A., Briel, U.G. 2004, ApJ, submitted

Krivonos, R.A., Vikhlinin, A.A., Markevitch, M.L., Pavlinsky, M.N. 2003, AstL, 29, 425

Kull, A., Böhringer, H. 1999, A\&A, 341, 23

Mahdavi, A., et al. 2000, ApJ, 534, 114

Neumann, D.M., Arnaud, M., Gastaud, R., et al. 2001, A\&A, 365, L74

Neumann, D.M., Lumb, D.H., Pratt, G.W., Briel, U.G. 2003, A\&A, 400, 811

Ponman, T.J., Sanderson, A.J.R., Finoguenov, A. 2003, MNRAS, 343, 331

Roettiger, K., Stone, J.M., Mushotzky, R.F. 1998, ApJ, 493, 62

Schuecker, P., Finoguenov, A., Miniati, F., Böhringer, H., Briel, U.G. 2004, A\&A submitted [arXiv:astro-ph/0404132]

Voit, G. M., Balogh, M.L., Bower, R.G., Lacey, C.G., Bryan, G.L. 2003, ApJ, 593, 272

\section{Discussion}

ETTORI: What are the implications of the entropy and pressure structure you reported to the mass measurements using the equation of hydrostatic equilibrium?

Finoguenov: The reported features could be segregated on those exhibiting pressure variations as due to the shock propagation and on those that exhibit only the entropy variation. In the former case, the formal mass measurements returns different values inside and outside the shock. E.g. in A754 the temperature in the outskirts experiences a transition from $5 \mathrm{keV}$ to $10 \mathrm{keV}$. The smaller total mass that would be measured using the $5 \mathrm{keV}$ temperature corresponds to a pre-merger state of the cluster, while the 10 $\mathrm{keV}$ temperature would correspond to a post-merger state of the cluster. An important note is that X-rays do not trace the total mass, but the virialized mass. As it becomes evident from other talks presented at this conference, the difference in the definition of a cluster is especially important in the comparison between clusters selected with weak lensing and X-rays. In the latter case, and in general, the use of the pressure to measure the total mass has certain advantages over using an X-ray image. However, an additional measure of the cluster virial temperature is required in the mass estimates and this task, as demonstrated in Silvano Molendi's contribution, is not trivial for current X-ray observatories.

WANG: What about the use of metallicity to study the substructure?

Finoguenov: Using metallicity as a tracer suffers from a poor statistics available at the moment and compared to the use of entropy achieves a much cruder spatial resolution. In most cases where it becomes possible, the entropy structure and metallicity structure are highly correlated. The level of chemical enrichment, on the other hand, sheds light on the origin of the low-entropy gas. 